\title{
Ouarsenis : Langue et sociolinguistique
}

Salem Chaker et Saïd Chemakh

\section{(2) OpenEdition}

Journals

Édition électronique

URL : https://journals.openedition.org/encyclopedieberbere/2855

DOI : 10.4000/encyclopedieberbere.2855

ISSN : 2262-7197

\section{Éditeur}

Peeters Publishers

\section{Édition imprimée}

Date de publication : 2 juin 2013

Pagination : 5946-5958

ISBN : 978-2-7584-0194-0

ISSN : 1015-7344

\section{Référence électronique}

Salem Chaker et Saïd Chemakh, « Ouarsenis : Langue et sociolinguistique », Encyclopédie berbère [En ligne], 36 | 2013, document 041, mis en ligne le 12 mars 2021, consulté le 17 février 2022. URL : http:// journals.openedition.org/encyclopedieberbere/2855; DOI : https://doi.org/10.4000/ encyclopedieberbere.2855

Ce document a été généré automatiquement le 17 février 2022.

(c) Tous droits réservés 


\title{
Ouarsenis : Langue et sociolinguistique
}

\author{
Salem Chaker et Saïd Chemakh
}

\section{NOTE DE L'ÉDITEUR}

[Cette notice a bénéficié de la relecture attentive et des suggestions J. Desanges et de K. Naït-Zerrad ; nous les en remercions vivement.]

1 L'étymologie berbère de l'oronyme Ouarsenis est transparente : il s'agit d'un syntagme composé de war, morphème privatif "sans" + (s-)nnig, préposition locative " au-dessus " $+-s$ « lui » (aff. pers. $3^{\mathrm{e}}$ pers. sing.), avec affaiblissement de la vélaire $[\mathrm{g}]$ en semi-voyelle palatale [y] (API [j]), caractéristiques des parlers de la région, puis vocalisation en [ī] long, selon un processus d'ailleurs largement attesté dans divers parlers berbères contemporains :

war sənnig-s > war sənniy-s > Warsənnīs = « Sans rien au-dessus de lui »

2 Cette dénomination fait évidemment allusion à la haute altitude du massif montagneux - il culmine au pic Sidi Amar à $1.985 \mathrm{~m}$-, que l'on voit des plaines du Chélif jusqu'à celles du Sersou.

3 Dans l'Antiquité, les sources latines le mentionnent deux fois, chez Pline l'Ancien (XIII, 95) et, trois siècles plus tard, chez Ammien Marcellin (XXIX, 5, 25), sous la forme $A n c(h)$ orarius, qui présente une très vague ressemblance avec le nom moderne, mais une étymologie latine est la plus plausible (cf. O40, note complémentaire de J. Desanges). Les sources arabes médiévales le désignent généralement sous la forme: Wanšarī̌s ("Ouancharich"), plus rarement waršenis (Ibn Khaldoun, Histoire des Berbères, notamment : I, 248 ; IV, 221). 


\section{Données sociolinguistiques}

L'enquête menée par E. Doutté et E.-F. Gautier en 1913 rapporte un fait significatif quant au processus d'arabisation de la région et à la déperdition de la langue berbère. Selon l'administrateur-adjoint de la commune mixte de l'Ouarsenis, le berbère était en nette régression, et même en voie de disparition; dans son courrier du 27 avril 1911 à Doutté \& Gautier, il en explicite la principale cause qui est, pour ses informateurs, d'ordre économique : les berbérophones de la montagne entretiennent des relations d'échanges commerciaux denses et régulières avec les arabophones des piémonts et plaines. Il précise (Doutté \& Gautier, p. 82) que :

«Ils sont ainsi arrivés, en deux générations, à délaisser d'abord au dehors puis dans leurs relations familiales, leur dialecte, au point, me disait un homme de trente ans, "que nous ne savons plus désigner la nourriture en kabyle".»

Plus loin, il note: «L'épithète de "Kabyle" est même devenue dans leur bouche un terme méprisant ».

Ce qui confirme un sentiment d'infériorité linguistique, voire de déconsidération sociale.

Il y a un siècle, Doutté \& Gautier (1913) donnaient comme berbérophones, en totalité ou en partie, les points suivants (tableaux des pages 118-121 ; voir carte ci-dessous) :

Commune de Djendel : Djebel Louh (= Hamza, Zbala, Ouled-Mahdi en partie)

Commune de Theniet El-Had : Haraoua et Tighzert.

Commune du Cheliff : Beni Boudouane (= Beni Rached : disparition récente)

Commune de l'Ouarsenis : Tikerdjichet, Bethaïa (en partie), Beni Bou-Khannous.

A. Basset dans son Atlas des parlers berbères (Algérie. Territoires du Nord) de 1936/1939 confirme l'existence de deux zones berbérophones dans l'Ouarsenis, l'une au nord de Bordj Bounaama (ex-Molière), l'autre au nord-est de Theniet El-Had, et mentionne :

Commune de Djendel : Djebel Louh (= Hamza, Zbala et Oued-Mahdi) ;

Commune de Theniet El-Had: Haraoua (= Beni Yala, Chekaïmïa, Ouled

Abderrahmane, Amrouna); Tighzert (= Saada, Younes, Sebaa);

Commune du Cheliff : Beni Bou-Khannous (= Ouled Aïssa, Layayda); Beni Bou Attab

(= Ikhenchoufen, Beni Djertin, Beni Bou Settour, Tarwa Maamer).

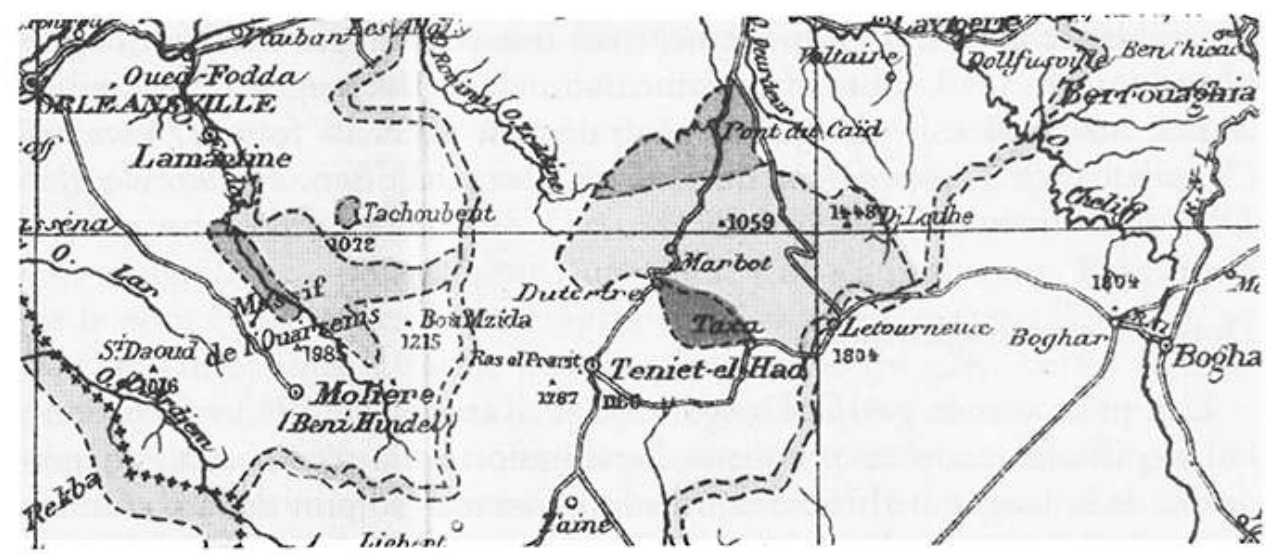

Fig. 1 : LE BERBÈre DANS LE MASSIF DE L'OUARSENIS, D'APRÈS DOUTTÉ \& GAUTIER, 1913. [MOLIÈRE = BORDJ BOUNAAMA ; MARBOT = TARIK IBN ZIAD ; LETOURNEUX = DERRAg ; VOLTAIRE = AIIN LECHIAKH] 


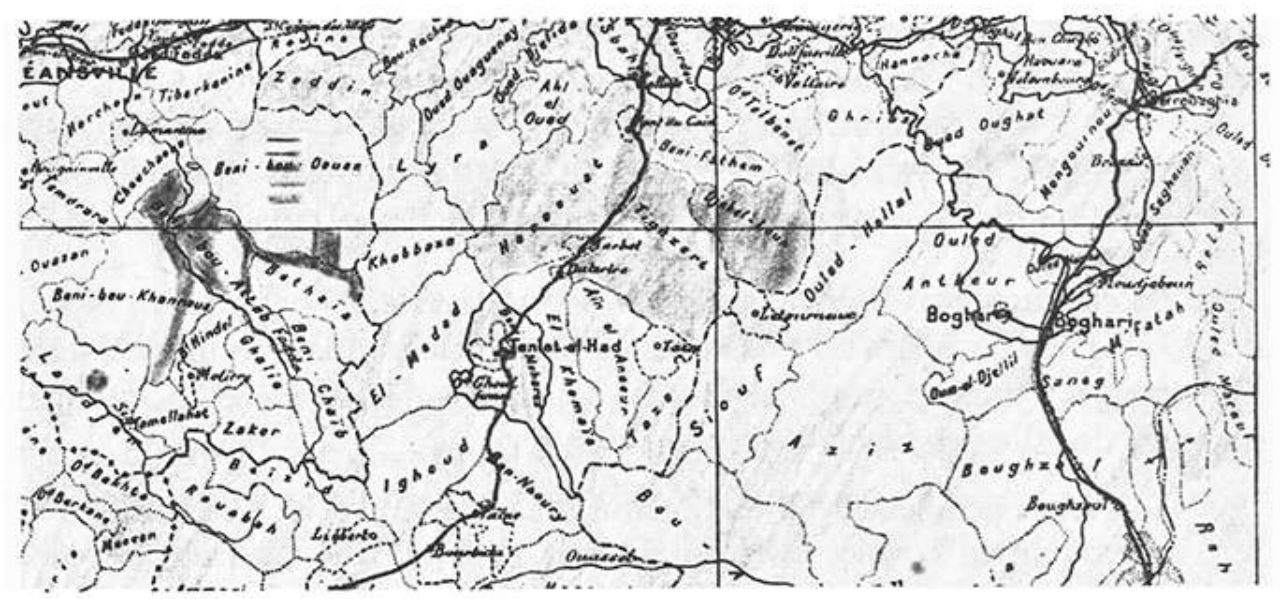

Fig. 2 : LE BERBÈre DANS LE MASSIF de I'OUARSENIS. SERVICE CARTOgRAPHIQUE DU GOUVERNEMENT GÉNÉRAL DE L'ALgÉRIE, 1922.

Les différences entre les deux listes correspondent sans doute plus à des redécoupages administratifs (sur les communes du Cheliff et de l'Ouarsenis) et à des fluctuations ou changements de dénominations qu'à une réelle évolution de la situation linguistique sur le terrain. En tout état de cause, on retiendra plutôt les données d'André Basset car elles reposent sur une véritable enquête linguistique de terrain, menée dans le cadre de son Atlas, alors que celles de Doutté \& Gautier, plus anciennes, résultent d'une enquête par questionnaire - certes systématique, mais néanmoins indirecte - conduite à travers le réseau des administrateurs et maires des communes de l'Algérie du Nord.

En 1936, Paul Beltzung, administrateur-adjoint de la commune mixte de Cheliff rédige un rapport, transmis au Gouvernement Général, où il notait que l'Ouarsenis est peuplé de huit douars regroupant près de 45.000 habitants pas tous d'origine arabe ; il signale notamment que :

« À l'encontre des autres groupes berbères de l'Algérie, l'Ouarsenis a de bonne heure été pénétré par la domination arabe.» (p. 3).

«Au contact de l'envahisseur, ils [les berbérophones] perdirent leur mœurs et leur langue. Par contre les Béni-Boudouane, qui, par la suite de leur isolement et de leur éloignement de tout centre étranger ont gardé plus longtemps un cachet particulier, et même leur langue... » (p. 4).

11 En 1972, Dj. Sari, qui soulignait «le surpeuplement de la zone culminante » du massif ne fait aucune mention de l'usage du berbère. Dans son étude de 1971, il mentionnait que :

"L'usage du berbère était très limité dans l'entendue de la chaîne [...] le souvenir des dialectes berbères, même par les vieilles personnes, a complètement disparu... ».

12 A la même période, J. Vignet-Zunz (1972/1974, Chap. II/6) considérait que le berbère était éteint dans la région, information que reprenait L. Galand dans sa chronique des études berbères (1979, réf. 1083, p. 141, mais aussi réf. 948, p. 126).

Les troubles de la seconde moitié du vingtième siècle ont contraint beaucoup de villageois berbérophones du massif à s'installer dans les villes limitrophes. L'apparition d'un maquis communiste en 1955, les opérations de l'armée française, ainsi que l'installation d'un maquis de l'OAS, ont poussé certains habitants à déserter leur village (cf. Boudiba 2003; Sudry 2008). Dans les années 1970, l'exode rural s'est accéléré à la suite de la politique du gouvernement algérien: l'essentiel des investissements 
d'infrastructures de l'Etat ayant été concentré sur les villes alors que les zones rurales, et surtout de montagnes, ont été largement délaissées. Les troubles des années 1990 les maquis islamistes étaient particulièrement actifs dans la région - ont de nouveau contraint beaucoup de villageois à abandonner le massif pour se réfugier dans les villes voisines.

Fait insolite, les descendants des immigrants kabyles, nombreux dans la région depuis les années 1920 (Theniet El-Had...), continuent à utiliser la langue berbère, alors que la plupart des berbérophones de l'Ouarsenis préfèrent l'arabe, allant parfois, jusqu'à nier leur origine berbère.

C'était là du moins la situation qui prévalait jusqu'aux années 1990. En fait, et malgré les affirmations ou constats ( ?) de Sari $(1971,1972)$ et de Vignet-Zunz (1972/1974), on restera très circonspect sur la question de la survie du berbère dans la région : prendre acte de sa disparition serait sans doute imprudent. On n'oubliera pas que la période à laquelle ont été menés ces travaux était marquée en Algérie par une vigoureuse politique d'arabisation et un contexte politique global autoritaire et très anti-berbère ${ }^{1}$; de plus, aucun de ces observateurs n'était berbérisant ou berbérophone. Il ne serait pas étonnant que l'amélioration du statut institutionnel et symbolique de la langue berbère en Algérie ait pu amener une partie des habitants de la région à revendiquer leurs racines berbères, voire à assumer leur berbérophonie jusque là honteuse et soigneusement cachée : on connaît de nombreux cas similaires de berbérophonie niée que l'on a vu "resurgir" lorsque les conditions sont devenues plus favorables, y compris parmi les locuteurs des grands dialectes berbères (chaoui notamment). Précisons également que le recensement algérien de la population de 1966, le seul qui ait comporté la rubrique « langue maternelle", n'est en l'occurrence d'aucun secours car les résultats qui en ont été publiés sont globalisés au niveau des wilayates (départements), alors que dans le cas d'espèce, il faudrait pouvoir disposer des résultats par communes - qui existent certainement, mais qui ne sont pas publics. Des enquêtes de terrain précises méritent donc d'être menées sur ce point dans la région et l'on évitera toute conclusion péremptoire et définitive ${ }^{2}$.

Il convient aussi de souligner que si l'usage de la langue berbère paraît très menacé dans l'Ouarsenis, elle y demeure très fortement présente dans la toponymie.

\section{Données linguistiques}

17 La variété de berbère de l'Ouarsenis fait partie de l'ensemble «zénète* » dans la classification du R. Basset $(1887$, p. 3), qui qualifie même les parlers locaux de «zénatia » (cf. son ouvrage de 1895). Cette dénomination/ classification n'est pas sans poser problème et doit être reçue avec circonspection :

- Il semble bien que ce sont les savants français (surtout René Basset) qui dénommaient ainsi le berbère de l'Ouarsenis. Il ne paraît pas du tout établi que les populations locales aient jamais usé du mot znatiya ou taznatit, du moins dans cette région; on sait au contraire qu'elles utilisaient le terme "kabyle". Rappelons que la dénomination "Kabyle" / (langue) "kabyle", (ləqbayəl / țaqbaylit > haqbaylit > aqbaylit), issue de l'arabe qabā'il, "tribus", était très largement employée, au moins jusqu'à la fin du XIX ${ }^{e}$, par les Arabophones citadins pour désigner toutes les populations berbérophones du nord de l'Algérie, en référence à la forme de leur organisation sociale en tribus. Cet usage est même très ancien et remonte aux premiers siècles de la conquête arabe, alors que les Arabes installés en Afrique du Nord 
étaient tous des citadins ; cet usage « large » se retrouve notamment dans le Raw لزوض المفر طاس) de 'Alī ibn 'Abd Allāh Ibn Abī Zar' al-Fāsī (début du XIVe siècle). Sur le sujet, on trouvera des développements très précis chez l'arabisant dialectologue Philippe Marçais (1954, p. 24-27). René Basset lui-même emploie d'ailleurs expressément le terme kabyle pour désigner tous les parlers berbères de l'Algérie du Nord (1890, p. 1).

- La dénomination et la classification paraissent en réalité fondées avant tout sur les grandes classifications géographiques et tribales des historiens arabes médiévaux: les Zénètes couvraient une large bande oblique Nord-Ouest / Sud-Est correspondant essentiellement aux hautes plaines et régions steppiques continentales, de l'Est du Maroc jusqu'au Djebel Nefoussa* ;

- Il existe certes un ensemble de critères linguistiques qui permettent de poser un ensemble « zénète » (cf. Chaker 1972 ; Kossman 1989, 1995a \& 1995b) ; mais il convient de souligner que ces critères linguistiques sont quelque peu fluctuants et pas toujours nettement discrimants par rapport au reste du berbère. Et dans le cas de l'Ouarsenis, sur la base des données disponibles, ils ne semblent s'appliquer que très partiellement (cf. infra). Les principales spécificités linguistiques de l'Ouarsenis sont néanmoins à rapprocher plutôt de celles des parlers du Chenoua*, du Mzab*, de Ouargla*...

18 Dans son Etude sur la zénatia de l'Ouarsenis et du Maghreb central (1895), R. Basset confirme son classement du parler de l'Ouarsenis parmi les dialectes «zénètes ", en précisant dans sa préface (p. I), que si :

«... plusieurs de ces dialectes berbères se maintiennent intacts, protégés par les montagnes, le désert ou l'hérésie [ibadisme], il en est d'autres qui, privés de ces appuis, s'éteignent de jour en jour, supplantés par l'arabe. Il est donc urgent de les recueillir avant qu'ils aient totalement disparus ".

19 Le berbère de l'Ouarsenis est donc l'un de ces petits parlers résiduels du centre-ouest algérien (Achacha, Beni Halima, Beni Menacer...) qui sont des lambeaux survivant du grand ensemble socio-historique, peut-être linguistique, zénète (R. Basset 1887, p. 3-4).

Il ne semble pas qu'il existe de travaux descriptifs d'ensemble du berbère de l'Ouarsenis depuis l'étude de R. Basset (1895). La grande misère de la documentation relative à la région est confirmée par les recensions bibliographiques systématiques de l'Annuaire de l'Afrique du Nord (Galand: période 1954-1979; S. Chaker: période 1980-1991; Cl. Brenier-Estrine: période 1992-1998) et la somme bibliographique de L. Bougchiche (1997) : elles ne font état que d'un nombre infime de références consacrées à ce parler, moins de cinq au total, depuis le début des études berbères.

21 Les données linguistiques présentées ici sont donc toutes issues de la monographie de R. Basset (1895), travail déjà très ancien, souvent peu précis et sujet à caution, notamment dans ses notations phonétiques qui sont assez approximatives. On détecte du reste des fluctuations et incertitudes tant phonétiques que grammaticales lorsqu'on compare ses textes du Loqmân berbère (1890) et les matériaux de son Etude (1895). Fluctuations qui pourrait aussi être dues au fait qu'il existe (ou existait) des différences sensibles, à la fois phonétiques et lexicales entre les deux zones berbérophones de l'Ouarsenis : les données collectées par A. Basset dans son Atlas (1936/1939) donnent par exemple :

22 - dans le bloc de Theniet El-Had (Haraouat, Tighzert, Djebel Louh) :

$y i s$, « cheval » et $[\theta a] g m a r \theta$, « jument » (avec $[\mathrm{g}]$, spirante médio-palatale comme en kabyle)

23 - dans le bloc de Bordj Bounaama (ex-Voltaire : Beni Bou Khannous, Beni Bou Attab) : 
$a \varepsilon^{a} u \delta i$, « cheval » et $[\theta A]$ yTAr $\theta$, « jument » (avec semi-voyelle palatale $\left.[y]\right)$.

Les dernières données linguistiques connues et publiées sont donc les quelques matériaux lexicaux (et phonétiques) fournis par A. Basset dans son Atlas (1936/1939).

\section{Phonétique-phonologie}

- L'Ouarsenis présente un système vocalique ternaire, tout à fait classique : /a, i, u/, sans distinction de durée, et un schwa ([ə]) non phonologique; les semi-voyelles /w/ et /y/ se distinguent des voyelles correspondantes, /u/ et /i/.

26 - Si l'on se fie aux notations de R. Basset, on constate une tendance, elle aussi classique, à l'ouverture de la vélaire $/ \mathrm{u} /(>[\mathrm{o}]):[\theta$ aməțtu $\theta]>[\theta$ aməțtu $\theta]$ en contexte emphatique ou emphatisant. Sous l'influence des emphatiques, on note ainsi la transformation régulières des schèmes en [o] : /awəḍ/ > [awod]], /asəmmiḍ/ > [asommiḍ].

27 - Le parler connaît le phénomène de spirantisation* (affaiblissement du mode d'articulation), largement attesté dans les parlers berbères du nord, de la Libye au Nord du Maroc, mais uniquement pour les occlusives dentales et les palato-vélaires :

$/ \mathrm{t} />[\theta] ; / \mathrm{d} />[\delta] ; / \mathrm{d} />[\delta]$

\begin{tabular}{|c|c|}
\hline - taməțtut > $\theta$ ATəțtu $\theta$, “femme" & - adrar > a $\delta$ rar, "montagne" \\
\hline - tafukt> $\theta f u y \theta$, "soleil" & - itri> iӨri, "étoile" \\
\hline - adəf>a > $\delta$ f, "entrer/pénétrer" & - abrid> abriס, "chemin" \\
\hline - taduft> $\theta a{ }^{\delta} u f \theta$, "laine" & - $a d u>a{ }^{\delta} u$, "vent" \\
\hline
\end{tabular}

Comme dans de nombreux parlers de la région mais aussi des parlers plus éloignés (chaoui, tamazight du Maroc central...), la dentale sourde /t/ $>[\theta]$ peut connaître une accentuation de ce processus d'affaiblissement et évoluer jusqu'à la laryngale [h], voire zéro, dans certains morphèmes de haute fréquence, notamment les pronoms indépendants, les indices de personne et les affixes personnels du verbe :

- nahnin, "eux", / nahnint "elles" (cf. kabyle : nitni-n / nitənti).

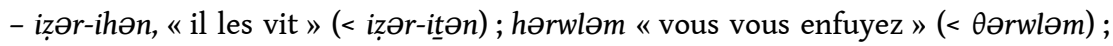

әzzarəт, « vous voyez » (< Өəzzarem).

En revanche, le parler ne semble pas connaître le phénomène d'affriction de la dentale tendue $/ \mathrm{tt} /\left(>\left[\mathrm{tt}^{\mathrm{s}}\right]\right.$, largement répandu en kabyle et dans une partie de l'Algérie centrale (Chenoua...).

$31 / \mathrm{g} />[\mathrm{y}]$ ou $[\check{z}]$

\begin{tabular}{|l|l|l|}
\hline $\mathrm{g}>\mathrm{y}$ & $-\operatorname{argaz}>$ aryaz, "homme" & \\
\hline & - aglim > aylim, "peau" & \\
\hline & - tagmart > Aaymar $\theta$, "jument" & \\
\hline
\end{tabular}




\begin{tabular}{|c|c|c|}
\hline$g>\check{z}$ & 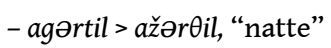 & - anəbgi > anuži, "invité" \\
\hline & - agənna > ažənna, "ciel" & - gar/gər > žar, "entre" (prép.) \\
\hline
\end{tabular}

\begin{tabular}{|c|c|}
\hline - kəyy/kəčč > šəkk, toi (masc.) & $-k \partial m>$ šəm, "toi" (fem.) \\
\hline 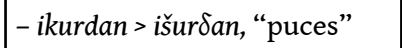 & 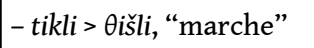 \\
\hline - kra > šra, "quelque (chose)" & \\
\hline
\end{tabular}

Il ne semble pas exclu que / $\mathrm{k}$ / puisse parfois évoluer vers la spirante médio-palatale, du moins si l'on accorde foi aux notations de R. Basset, qui donne a a ab, "renard", transcrit en arabe, ce qui laisse supposer plutôt un [k] ([akab]) qu'un [x] ou [š].

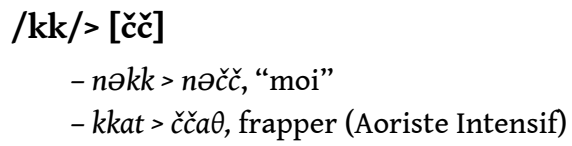

\section{Morphologie et syntaxe}

34 - Comme dans les autres parlers « zénètes ", on relève une tendance nette à la chute de la voyelle initiale dans les thèmes nominaux de structure syllabique initiale $-\mathrm{CvC}$ :

$$
\begin{aligned}
& \text { dad, "doigt" (< a dad) } \\
& \text { fus, "main" (< afus) } \\
& \text { fud, "genou" (< afud). } \\
& \text { yug / yuy, "boeuf'(< ayug) }
\end{aligned}
$$

35 - L'état d'annexion (EA) des noms masculins singuliers (initiale $u$-) ne semble pas toujours marqué ; on relève dans les textes de R. Basset :

$i$-zru -t uryaz = «l'homme le vit »

il-vit -le homme (+EA)

mais aussi :

$i$-zrru -t akab = « le renard le vit »

il-vit -le renard (+EL)

37 Sur ce cas, on ne peut cependant exclure une erreur de R. Basset ou une hésitation individuelle de son informateur et on n'accordera qu'une confiance limitée à ce témoignage..

38 - Le déictique de proximité post-nominal est, comme en kabyle, - $a$, alors que l'on rencontre généralement $-u$ dans les autres parlers zénètes :

- aryaz-a, "cet homme"

39 - Comme en kabyle, les affixes personnels suffixes du nom ("possessifs") se présentent, pour les formes du singulier, sous la variante (primitive) courte (R. Basset 1895, p. 34) :

- iw, "ma, mon, mes"

$-ə k$, “ta, ton, tes" (possesseur masc.) 
- im, “ta, ton, tes" (possesseur fém.)

- is, "sa, son, ses"

\section{Système verbal}

Dans son Etude, R. Basset présente le système binaire classique aoriste/prétérit tel qu'on le concevait à son époque. Il est donc difficile de se faire une idée du fonctionnement du verbe de l'Ouarsenis, d'autant que les textes qu'il fournit (1890) sont extrêmement brefs et non spontanés puisqu'il s'agit de traductions de fables. On peut néanmoins inférer de ces matériaux un certain nombre de données :

43 - Le système est bien, comme c'est largement le cas dans le reste du berbère Nord, ternaire : Aoriste / Aoriste intensif / Prétérit, avec un allomorphe de Prétérit négatif. Il n'est pas possible de vérifier s'il y existe un thème d'Aoriste Intensif négatif, connu dans plusieurs autres parlers « zénètes » (Kossman 1989).

44 - Sous réserve des incertitudes des notations de R. Basset, le thème d'Aoriste nu semble, comme dans de nombreux autres dialectes berbères (tamazight, kabyle...), pouvoir être utilisé dans le récit pour marquer l'enchaînement des actions (cf. texte cidessous) :

$i z ə r-i h ə n$ ukab $=«[. .$.$] un renard les vit [. .]$.

45 - Le préverbe ad de l'Aoriste parait avoir les mêmes emplois qu'en kabyle, à la fois temporels (futur) et modaux ; il est défini comme « futur » par R. Basset.

46 - Il semble alterner (?) devant l'Aoriste avec un préverbe da, bien connu ailleurs (Tamazight du Maroc central) comme préverbe de l'Aoriste Intensif, marquant la durée ou la concomitance :

ihwa da isw = « il eut envie de boire » (on attendrait : *ihwa ad isw)

47 - Basset donne des exemples dans lesquels le verbe ili, "être/exister" semble fonctionner comme auxiliaire de concomitance/durée, que l'on rencontre aussi dans d'autres parlers (Mzab, Ayt Seghrouchen...) :

Llan təttən, ils étaient ils mangeaient $=$ « ils étaient en train de manger ».

48 - On relève l'auxiliaire de passé révolu ittuy (+ Prétérit), connu depuis l'Est du Maroc (Ayt Seghrouchen) jusqu'au Mzab et Ouargla, en passant par la Kabylie orientale.

49 - Le parler présente l'un des traits spécifique de la morphologie verbale des parlers zénètes (Chaker 1972; Kossmann 1995a \& 1995b) : au thème de Prétérit, les verbes bilitères à voyelle finale alternante ont un $/ \mathrm{u} /$ à la $3^{\mathrm{e}}$ pers. du masc. sing. et un $/ \mathrm{i} / \mathrm{aux}$ $2^{\mathrm{e}}$ et $3^{\mathrm{e}}$ pers. du pluriel :

zər, voir

zrix, "j'ai vu”

iz̧ru, "il a vu" 
təẓra, "elle a vu" (?)

nəzra, "nous avons vu

təzrim "vous avez vu"

təẓrimt "vous avez vu"

zrin, "ils ont vu"

zrint, "elles ont vu"

50 - La négation verbale a la forme $u$ - ou wala - ; le second élément (š ou šəy) ne semble pas obligatoire :

wala təxdimt «tu n'as pas fait »

\section{Lexique}

51 Outre le lexique fourni par R. Basset (1895), E. Destaing mentionne 1.675 notations dans son Dictionnaire français-berbère (1914) pour les Matmatas de Djendel et 31 pour les Haraouas. On dispose également des données de l'Atlas d'A. Basset (1936/39) pour les noms d'animaux domestiques.

52 Pour ce qui est des textes, il n'en existe que cinq, très courts (en réalité quatre), collectés par R. Basset: quatre dans le Loqmân berbère $\left(1890\right.$; fables $n^{\circ} 9$, p. $58 ; n^{\circ} 17$, p. $83 ; n^{\circ} 30$, p. $93 ; n^{\circ} 30$, p. 128) et un dans son Etude de 1895 (identique à la fable $n^{\circ} 9$ de 1890).

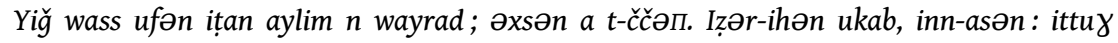
iddər wayrad, a lukan əzzarəm Təxalibən-is, hərwləm! (R. Basset, 1890, fable ${ }^{\circ} 30$, p. 128).

«Un jour des chiens trouvèrent une peau de lion; ils voulurent la manger. Un renard les vit et leur dit: "Ah, lorsque le lion était vivant, si vous aviez vu ses griffes, vous vous seriez enfuis!».

53 Sur la base des données disponibles, on peut donc constater que si le caractère « zénète » du parler est bien confirmé par plusieurs traits de morphologie verbale et nominale, éventuellement de phonétique - mais ce type de critères paraît extrêmement fragile et peu pertinent -, par d'autres aspects, il se rapproche plutôt du kabyle. Cette conclusion provisoire et prudente tendrait à rappeler que la classification linguistique des variétés du berbère demeure très problématique: le continuum berbère a certainement toujours été caractérisé par des transitions «douces» et le chevauchement des isoglosses. 


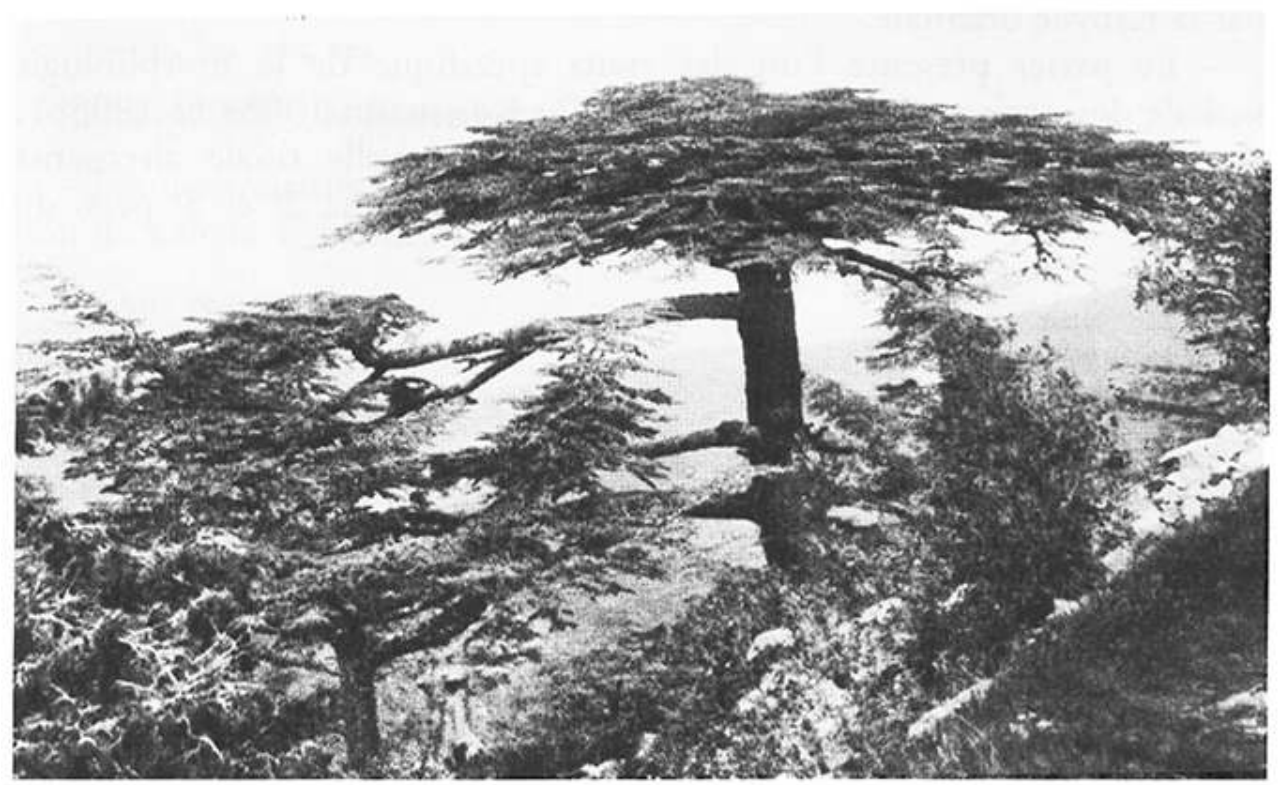

Fig. 3 : FORÊT DE CÈdRES DE THENIET-EL-HAD (BROCHURE TOURISTIQUE OFALAC, ALgER ; S.D., VERS 1930 (PHOTO GOUVERNEMENT gÉNÉRAL DE L'ALgÉRIE).

$54 \mathrm{Au}$ terme de cette présentation trop lacunaire, on ne peut que redire l'urgence d'enquêtes sociolinguistiques et linguistiques sur le terrain, qui auront pour objectif de vérifier à la fois la survie et la pratique de langue dans la région et de permettre une description plus consistante de cette variété de berbère.

\section{BIBLIOGRAPHIE}

AMMIEN MARCELLIN, Histoire de Rome, Livre XXIX, 5, 25.

Annuaire de l'Afrique du Nord, Paris, Editions du CNRS : « Langue et Littérature berbères : chroniques des études ", du vol IV, 1965 au volume XXXVIII, 1998 (par L. Galand, S. Chaker, Cl. Brenier-Estrine)..

BASSET A., 1936/1939 - Atlas linguistique des parlers berbères, Alger, Institut d'Études Orientales [+ cartes].

BASSET A. 1946 - « Enquête linguistique dans la zone nord du Chélif, Journal Asiatique, 1946-1947, p. 194.

BASSET A., 1952 - La langue berbère, 1969 (2 éd.), Oxford Université Press, London BASSET R., 1890 - Loqmân berbère, Paris, Leroux.

BASSET R., 1895 - Etude sur les Zenatia de l'Ouarsenis et du Maghreb Central, Paris, Leroux.

BELTZUNG P., Rapport Ouarsenis, in Archives du Gouvernement Général de l'Algérie, année 1936, Archives Nationales d'Outre-Mer, Aix-en-Provence, [consulté en juin 2011]. 
BERBRUGGER A., 1856/1857 - « Antiquités du cercle de Ténès », Revue africaine, vol. 5, p. 335-345 \& vol. 7, p. 182-83.

BOUDIBA M., 2003 - L'Ouarsenis. La guerre au pays des cèdres, Paris, L'Harmattan.

BOUGCHICHE L., 1997 - Langues et littératures berbères des origines à nos jours. Bibliographie internationale, Paris, Ibis Press.

CADENAT P., 1963 - « L'art rupestre préhistorique du département de Tiaret (Algérie) », Soc.

d'Etudes et de recherches préhistorique (Les Eyzies, Institut pratique de Préhistoire, Bull. $\mathrm{n}^{\circ} 13$, trav. de 1963), p. 34-51.

CHAKER S., 1972 - «La langue berbère au Sahara », Revue des mondes musulmans et de la Méditerranée, 11/1, p. $163-167$.

CHAKER S., 1993 - « Chenoua », EB, XII, p. 1902-1904.

CHAKER S. 1993 - « Chaoui”, EB, XII, p. 1875-1877.

CHAKER S., CHEMAKH S., NOUH A, 2010 - « Mzab - Mozabite : langue et littérature », EB, XXXII, p. $5181-5190$

DESTAING E., 1914 - Dictionnaire français-berbère, Paris, Leroux.

DOUTTÉ E. \& GAUTIER E.-F., 1913 - Enquête sur la dispersion de la langue berbère en Algérie, Alger, Jourdan.

GALAND L., 1979 - Vingt cinq ans d'études berbères, Paris, CNRS.

IBN KHALDOUN, Histoire des Berbères, Paris, 1925 (rééd.)

JULIEN Ch.-A., 1951/1978 (nombreuses rééditions) - Histoire de l'Afrique du Nord, Payot, 2 t. C'est l'édition algérienne (Alger, SNED, 1978) qui est citée ici.

KosSMANN M., 1989 - «L'inaccompli négatif en berbère ", Etudes et Documents Berbères, 6, 1989, p. 19-29.

KOSSMANN M., 1995a - « La conjugaison des verbes CC à voyelle alternante en berbère ", Etudes et Documents Berbères, 12, p. 17-33

KOSSMANN M., 1995b - «Les verbes à finale $i$ en zénète : étude historique », Etudes et Documents Berbères, 13, p. 99-104.

MARÇAIS Ph., 1954 - Textes arabes de Djidjelli, Paris, PUF (Publ. de la Faculté des Lettres d'Alger : XXVI).

PLINE l'Ancien, Histoire naturelle, Livre XIII, 95.

SARI Dj., 1971 - « L'équilibre économique traditionnel des populations de l'Ouarsenis central », ROMM, 9, p. 63-89

SARI Dj., 1972 - « Les populations de l'Ouarsenis central », Méditerranée, (2e série), 11/3-4, p. 89-117.

SUDRY Y., 2008 - Guerre d'Algérie : les oueds rouges de l'Ouarsenis, Paris, L'Harmattan.

VIGNET-ZUNZ J., 1972 - Homme de l'Ouarsenis : une communauté rurale d'Algérie, Thèse de Doctorat de $3^{\mathrm{e}}$ cycle, Paris, Université René-Descartes (M. Leroi-Gourhan, Dir.). Disponible sur microfiches : 1974, Paris, Institut d'Ethnologie (Archives et Documents), microfiche $\mathrm{n}^{\circ}$ 74.012.103. Cf. notamment Chap. II/6, "L'Ouarsenis : sa personnalité et l'hypothèse berbériste ». 
VIGNET-ZUNZ J., 1977 - « L’habitat rural dans l'Ouarsenis (Algérie) », Techniques et Cultures, 2, p. 27-78.

VIGNET-ZUNZ J., sous presse - Montagnes et savoirs en Méditerranée. Ouarsenis (Algérie), Jabal Al-Akhdar (Libye), Rif (Maroc), Alger, CNRPAH (\& Aix-en-Provence, IREMAM).

YACONO X., 1955 - La colonisation des plaines du Cheliff..., t. I \& II, Alger, Imp. Imbert.

\section{NOTES}

1. On rappellera que le début de la décennie 1970 correspond à l'apogée du régime de Houari Boumediene, issu du coup d'Etat du 19 juin 1965. Parti unique, contrôle absolu de l'espace et de l'expression publiques, répression systématique et expéditive des oppositions (assassinats politiques et détentions arbitraires...), politique d'arabisation systématique et affirmation officielle de l'identité arabe et musulmane de l'Algérie sont, avec l'étatisme économique poussé à l'extrême, les principaux traits de cette période, peu propice à l'expression de la berbérité et de quelque particularisme que ce soit.

2. S. Chaker a dans son entourage personnel des amis Kabyles émigrés dans la région (Theniet ElHad) qui lui ont souvent affirmé converser en berbère avec les gens du crû.

\section{INDEX}

Mots-clés : Algérie, Linguistique, Onomastique, Zénète 